\title{
PAPR Reduction in Coherent Optical OFDM System using Modified Sliding Norm Transformer
}

\author{
Liqaa A. Al-Hashime ${ }^{1}$, Sinan M. Abdul Satar ${ }^{2}$, Ghaida A. AL-Suhail ${ }^{3}$ \\ ${ }_{1,3}$ Department of Electrical Engineering, University of Basrah, Basrah, Iraq. \\ ${ }^{2}$ Department of Electrical Engineering, University of Technology, Baghdad, Iraq \\ leqaa.abdulsattar@gmail.com,sinansma@yahoo.com,ghaida_alsuhail@yahoo.com
}

\begin{abstract}
Orthogonal Frequency Division Multiplexing is one of the most important multiple carrier modulation format, which has many applications in wireless communication and optical communication. It is considered as an excellent method for fast optical communication inferable from its high spectral efficiency and its strength to path losses. Peak to average power ratio is regarded as one of the main problems that are experienced by the optical orthogonal frequency division multiplexing system, which directly affects the characteristics of the system. The current paper proposes an efficient technique to reduce Peak to Average Power Ratio by the modified Dursun's method or the so called modified discrete sliding norm transformer in coherent optical orthogonal frequency division multiplexing system for the first time to the base of our knowledge consequently. The proposed technique does not need to send side information to the receiver; also, it does not degrade bandwidth. This algorithm lessens Peak to Average Power Ratio in the optical coherent Orthogonal Frequency Division Multiplexing system to about $4.15 \mathrm{~dB}$ at complementary cumulative distribution function (CCDF) probability of $10^{-3}$ and improves the system performance.
\end{abstract}

Index Terms- Orthogonal Frequency Division Multiplexing (OFDM), Peak-toAverage Power Ratio (PAPR), Complementary Cumulative Distribution Function (CCDF), Modified Sliding Norm Transformer (MSNT), Coherent Optical OFDM(COOFDM).

\section{INTRODUCTION}

The OFDM system has gained vital importance as one of the multi-subcarrier technique which has many applications in wireless communication and optical communication. The optical OFDM is considered as an essential method for high-speed optical fiber communication and in the long haul because of its good spectral efficiency, high data rate in addition to its strong capability of anti-dispersion. To give an illustration of OFDM, it is a pattern of Multi Modulation (MM) carrier system where the signal stream is divided into blocks [1]. Each block is carried by a subcarrier which is different from the next block transmitted by different subcarrier at the same time without causing interference between them because they are orthogonal to one another. In other words, it means that they are independent of each other. This can be obtained by putting the carrier at the null as in figure (1). 


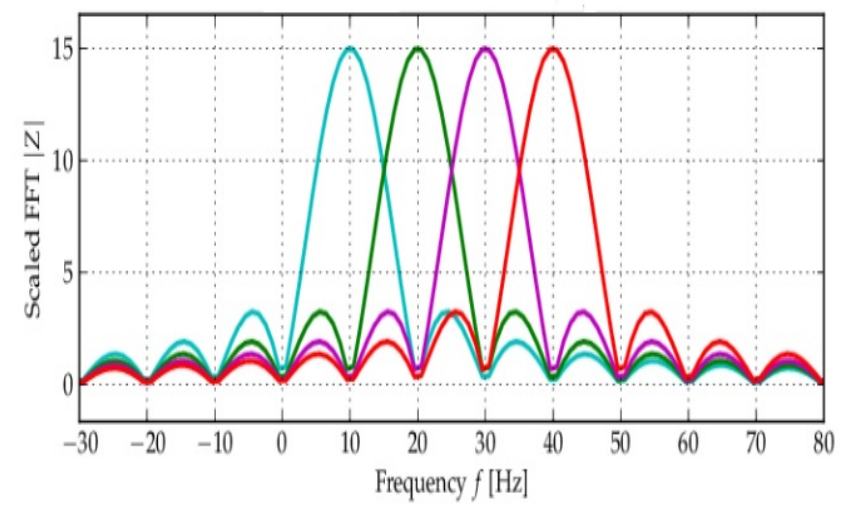

Fig. (1) FouR-ChanNEL BPSK OFDM Signal SPECTRUM[2]

The multicarrier transmitted signal is represented by [3]:

$$
\begin{gathered}
s(t)=\sum_{i=-\infty}^{\infty} \sum_{k=1}^{N_{s c}} c_{k i} \exp \left(j 2 \pi f_{k}\left(t-i T_{s}\right) f\left(t-i T_{s}\right)\right. \\
f_{k}=\frac{k-1}{t_{s}}, \Delta f=\frac{1}{t_{s}} \\
f(t)=\left\{\begin{array}{l}
1,\left(-\Delta_{G} \leq t \leq t_{s}\right) \\
0,\left(t \leq-\Delta_{G}, t>t_{s}\right)
\end{array}\right.
\end{gathered}
$$

Where $\mathrm{C}_{\mathrm{ki}}$ represents the ith information symbol at the kth subcarrier, $f(t)$ represents the waveform for the kth subcarrier, Nsc represents the Number of subcarriers, Ts represents the symbol period, $f_{k}$ represents the frequency of subcarrier, $\Delta f$ represents the subcarrier spacing, $\Delta G$ represents the guard interval, and ts is the observation period.

Nevertheless, a prima disadvantage of OFDM is superior PAPR. Hence, the influence of a high PAPR gets to be awful for the higher amount for subcarriers. Since in the worst case the PAPR for an OFDM symbol is equal to $10 \log 10 \mathrm{Nsc}$, where Nsc is the number of subcarriers. Furthermore, in optical OFDM which is a high data rate transmission, there is a need for the higher number of a subcarrier, so the PAPR reduction techniques are of specific concern[4].

Many techniques are used in order to reduce PAPR which could be classified into linear and non-linear categories. Likewise, linear techniques are classified into distortion and distortionless. Examples of distortion techniques are Clipping and Filtering[5], Windowing[6] and Compounding[7]. The distortionless techniques like, Selective Mapping Technique(SLM)[8], Partial transmit sequence(PTS)[9], inter-leave[10], Tone injection \& tone reversion(TI\&TR)[11], Active Constellation Excitation[12], Coding[13] and PreCoding[14]. While nonlinear techniques include Reduced Complexity Max Norm algorithm (RCMN), Modified Sliding Norm Transform (MSNT) and L2by3[15].

In nonlinear techniques, there is no need to send the side information to the receiver. So, the complexity of the system will be reduced, and there is no loss in data rate and bandwidth. This paper proposed to use Modified Sliding Norm Transformer (MSNT) which is the nonlinear technique to reduce PAPR in CO-OFDM for the first time to the best of our knowledge. This article is divided into many sections: The introduction is presented in section one, while section two is a review of the PAPR problem in OFDM, a review of MSNT. Section three is the result and simulation, and finally the discussion is presented. 


\section{PAPR Issue in OFDM}

In the OFDM system, high-speed incoming data are converted into low-speed symbols and a parallel format. The next stage converts the parallel symbols into a complex vector of size N.The baseband data are fed to the Inverse Fast Fourier Transformer(IFFT)stage in order to produce an(S0, $\mathrm{S} 1, \mathrm{~S} 2 \ldots \mathrm{SN}-1)$. The signal can be written as[1]:

$$
s(t)=\frac{1}{\sqrt{N}} \sum_{k=0}^{N-1} S_{k} e^{j \frac{2 \pi}{L N} k n} 0 \leq t \leq N T
$$

Where $\mathrm{N}$ represents the number of subcarriers, when the extensive range of signals from entirely different subcarriers is added, the signals with similar phases at an equivalent time instants can produce high amplitude peaks. Compared with the average signal power, the instantaneous power of those peaks is exceptionally high, making the electronic equipment of the transmitter work in the non-linear region that considerably degrades the system performance. Another critical point is the existence of a large number of severally modulated sub-carriers in the OFDM system, so the peak value of the system can be proved; as high, in comparison with the average of the whole system. The PAPR of the transmit signal is[16]:

$$
P A P R=\frac{P_{\text {peak }}}{P_{\text {avg }}}=10 \log _{10} \frac{\max _{k}\left[\left|s_{k}\right|^{2}\right]}{E\left[\left|s_{k}\right|^{2}\right]}
$$

Where Ppeak is the peak output power, and Pavg is the average output power. Minimizing $\max |x(t)|$, is the goal of all PAPR methods. For appropriate estimations of PAPR, the oversampling factor is utilized. The oversampling factor $\mathrm{L}=4$ gives adequate tests to catch persistent domain signal peaks. The oversampled is applied by adding zeros to the vector of the original information and then converting the signal from the frequency zone to the time zone. [17]. Cumulative distribution function (CDF) is a parameter used to discover the effectiveness of any PAPR technique. Commonly the Complementary CDF is used alternatively of CDF. Still yet the purpose behind such use is helping to determine the probability that PAPR of a definite data block transcends the given threshold. The CCDF for the signal sample having amplitude is given by[18].

$$
\operatorname{CCDF}(\operatorname{PAPR}(x[n]))=\operatorname{Prob}(\operatorname{PAPR}(x[n]>\operatorname{PAPR} 0))
$$

\section{A. Nonlinear Modified sliding Norm Transformer technique}

This technique is modified from Dursun's algorithm or the so called discrete sliding norm transformer (DSNT) that is mentioned and used for the first time in the wireless system. The L2-DSNT or Dursun's technique has been mentioned in[19]. This algorithm does not need any additional information to send to the receiver. In the transmitter, it needs to use three samples $\left(\mathrm{S}_{n-1}, \mathrm{~S}_{n}, \mathrm{~S}_{n+1}\right)$ and the parameter that has a major controlling is $(\alpha)$ for each sliding window, to determine the output sample[19]:

$$
y_{n}=\frac{s_{n}^{\#}}{\sqrt{\alpha+s^{2} n-1+s^{2} n+s^{2} n+1}}
$$

At the $(\mathrm{Rx})$ the inverse of L2_DSNT is:

$$
\mathrm{s}_{\mathrm{n}}=\left(\sqrt[2]{\mathrm{s}_{\mathrm{n}}^{2}}\right) \operatorname{sign}\left(\mathrm{y}_{\mathrm{n}}\right)
$$

For the modified sliding norm transformer MSNT which is first proposed and used to reduce PAPR in[16], then it is used in combination with clipping for reducing PAPR indirect detection optical orthogonal frequency division multiplexing DD-OOFDM[20]. This technique minimizes computational complexity by using only two samples $\left(\mathrm{S}_{n-1}, \mathrm{~S}_{n}\right)$ in the transmitter. Nonetheless, there are two parameters 
$\alpha$ and $\beta$, which are used to determine the required PAPR and power spectral density. The MSNT is defined as[16]:

$$
. . y_{n}=\left\{\begin{array}{cc}
s_{0} & n=0 \\
\frac{s_{n}}{\sqrt{a+b .\left|s_{n}\right|^{2}+\left|s_{n+1}\right|^{2}}} & n=1,2,3, \ldots N-1
\end{array}\right.
$$

In the receiver the inverse of the transform is:

$$
s_{n}=\left\{\begin{array}{c}
y_{0}, y_{n} \neq 0, n=0 \\
y_{n} \cdot \sqrt{\frac{a+\left|s_{n-1}\right|^{2}}{1-b \cdot\left|y_{n}\right|^{2}}} \\
0 \quad y_{n}=0
\end{array}\right.
$$

PAPR value will be changed depending on $(\alpha$ and $\beta$ ) values. PAPR is directly proportional to $\alpha$, where $\alpha$ changes from $0.1-1$ and inversely proportional to $\beta$, where $\beta$ is changed from $1-10$. This technique is not affected by the order of mapping [16].

\section{SYSTEM SETUP}

Figure (2) illustrates the plan of the proposed Optical OFDM system by utilizing the nonlinear MSNT algorithm. Fundamentally, the proposed system varies from the traditional optical OFDM in that there is MSNT in the (Tx) and Inverse MSNT in the (Rx). As appeared in Figure (2), the system comprises transmitter - optical channel -receiver, which will be discussed underneath.

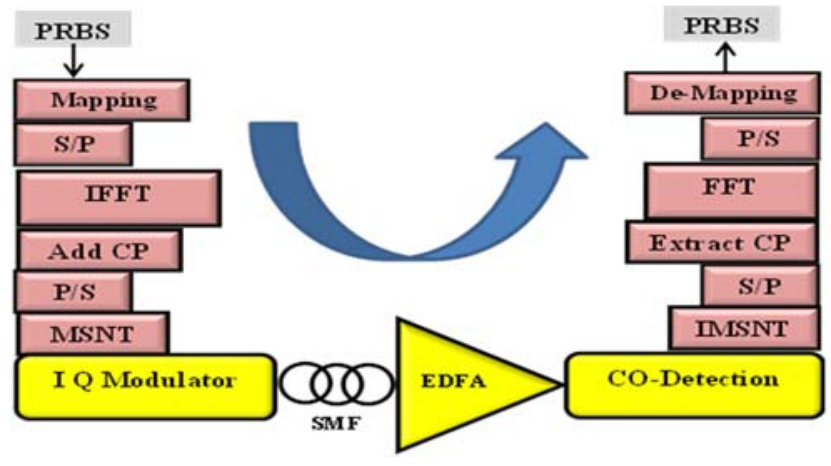

Fig. (2) Proposed Optical OFDM SYSTEM WITH MSNT

\section{A. OFDM Transmitter}

The pseudo-random binary sequence generates binary data at high speed. These data are arranged in symbols, and the number of bits in each symbol depends on the type of mapping. The mapping may be Binary Phase Shift Keying(BPSK), Quadrature Phase Shift Keying (QPSK) and Quadrature Amplitude Modulation (M-QAM), for example when using 4 QAM; there are two bits per symbol so the number of bits in symbol equals to $\log 2(\mathrm{M})$, where $\mathrm{M}$ equals to the order of Mapping. The complex symbol is then converted from serial to parallel in order to be modulated on the subcarrier, each symbol to a separate subcarrier, by using the inverse fast Fourier transform which converts the signal from the frequency domain to the time domain. The next step is to add the cyclic prefix in order to ensure that no intersymbol interference or inter-carrier interference happens when the signal passes through the optical channel. The step after adding the cyclic prefix in the transmitter part is to use an MSNT algorithm to minimize PAPR of the OFDM signal. When PAPR decreases, this will reduce the impact of nonlinear impairments on the signal. The last step in the transmitter is converting the OFDM signal from parallel to serial form to send it through the channel. 


\section{B. Optical Channel}

The Radio Frequency( RF) signal is converted to the optical signal using the IQ modulator. The real component (I) and the imaginary component (Q) of the output signal from the OFDM transmitter are sent to I/Q optical modulator that contains two lithium Niobate (LiNb) Mach-Zehnder modulators (MZM). The electrical signal is converted to an optical signal using (MZM) which modulates the signal on the optical carrier $193.1 \mathrm{THz}$ that is produced by Continues Wave Laser diode. The optical signal is then sent through the single mode fiber (SMF) with a dispersion compensating fiber (DCF) and an Erbium Doped Fiber Amplifier (EDFA) which is used to amplify the signal and to compensate the losses due to noise which happens during transmission. The last part of the optical channel is coherent optical detection - the CO-OFDM receiver which convertes the optical signal to the electrical signal using four photodetectors with a phase difference among them given by (90), in order to bring the OFDM signal back to the baseband electrical signal.

\section{OFDM Receiver}

In the receiver, the detected signal enters the inverse of nonlinear MSNT in order to reconstruct the signal that we sent. The cyclic prefix is removed in order to do (FFT) which converts the signal from time domain to frequency domain then the signal is converted to the serial form in order to do demapping and retrieval of the original signal.

\section{Discussion}

The simulation of the CO_OFDM system with PAPR reduction which is mentioned in this paper has used virtual photonic integrated transmission Maker program (VPI) with Matlab co-simulation to simulate the system. The OFDM coder and decoder with the MSNT algorithm for PAPR reduction were built all in Matlab (R2014a). The system setting is listed in Table 1.

TABLE 1. TYPE SIZES AND APPEARANCE

\begin{tabular}{|c|c|}
\hline \multicolumn{2}{|c|}{ Global Parameters } \\
\hline Bit Rate & $10 \mathrm{Gbps}$ \\
\hline Sample Rate & 40 Gbps \\
\hline Mapping Order & 4QAM \\
\hline Number of Subcarrier Nsc & 128 \\
\hline Bit per symbol BpS & 2 \\
\hline Cyclic Prefix CP & $1 / 8$ \\
\hline Over Sample & 4 \\
\hline \multicolumn{2}{|l|}{ Laser Parameter } \\
\hline \multicolumn{2}{|l|}{ Line Width for Laser } \\
\hline \multicolumn{2}{|l|}{ Laser Power } \\
\hline \multicolumn{2}{|c|}{ Optical Channel Parameter } \\
\hline Length of Single Mode Fiber SMF for one loop & $50 \mathrm{~km}$ \\
\hline Length of Dispersion Compensating Fiber DCF & $5 \mathrm{~km}$ \\
\hline Attenuation for SMF & $0.2 \mathrm{~dB} / \mathrm{km}$ \\
\hline Attenuation for DCF & $0.5 \mathrm{~dB} / \mathrm{km}$ \\
\hline Optical Amplifier Gain(EDFA) & $13 \mathrm{~dB}$ \\
\hline Optical Amplifier Noise Figure & $4 \mathrm{~dB}$ \\
\hline
\end{tabular}

First of all, we will investigate the effect of the factors $\alpha$ and $\beta$ on the PAPR value. Figure 3 shows that once $\alpha$ changes from $0.1-1$ and $\beta$ equals to 8 , the PAPR value can increase from $4.9 \mathrm{~dB}$ to 10.4 
$\mathrm{dB}$ when the probability of the given threshold is $10^{-3}$. As mentioned before, the PAPR value is directly proportional to the value of $\alpha$.

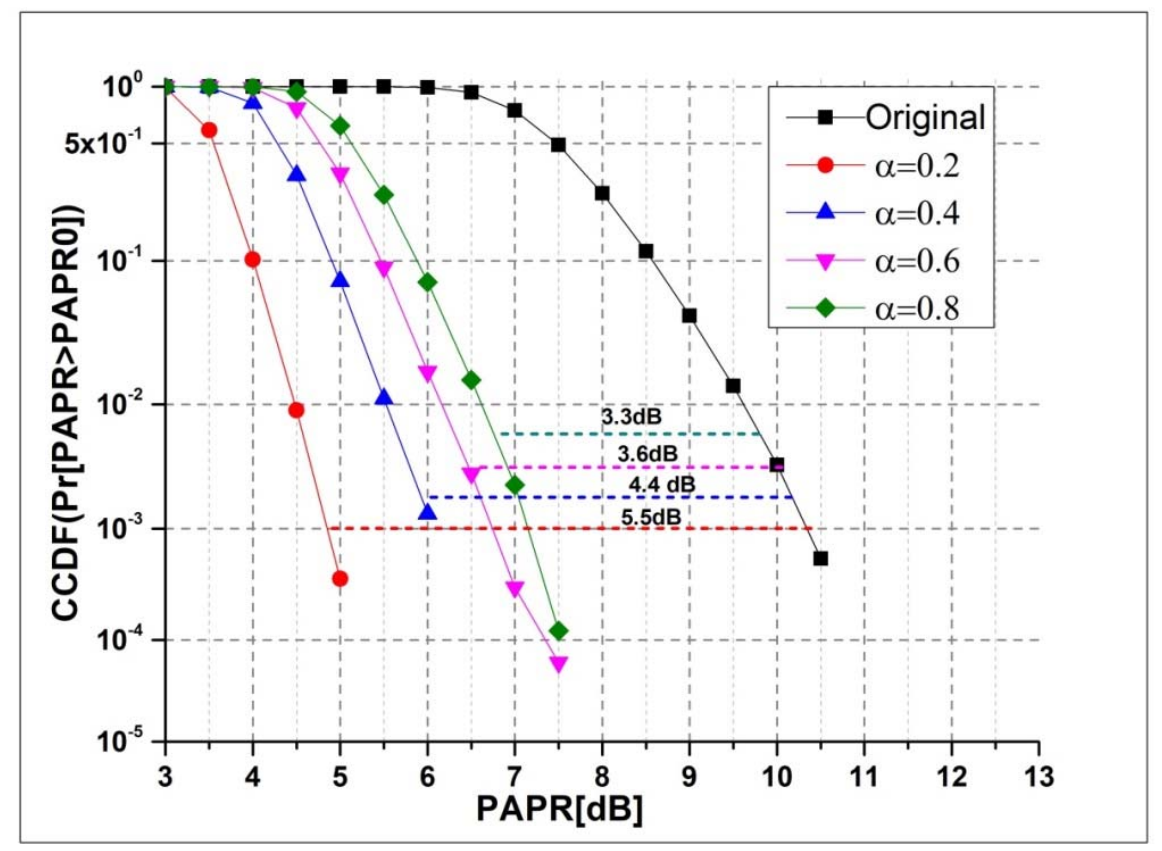

Fig.3. PAPR FOR DifFERENT VALUES OF A, B=8, NSC=128, 4QAM

Figure 4 shows the effect of changing $\beta$ from 1-10 and $\alpha$ takes a constant value of 0.2 on the value of PAPR. PAPR is inversely proportional with $\beta$, and when $\beta$ increases, PAPR decreases from $4.9 \mathrm{~dB}$ to $10.4 \mathrm{~dB}$, when the probability of the given threshold is $10^{-3}$.

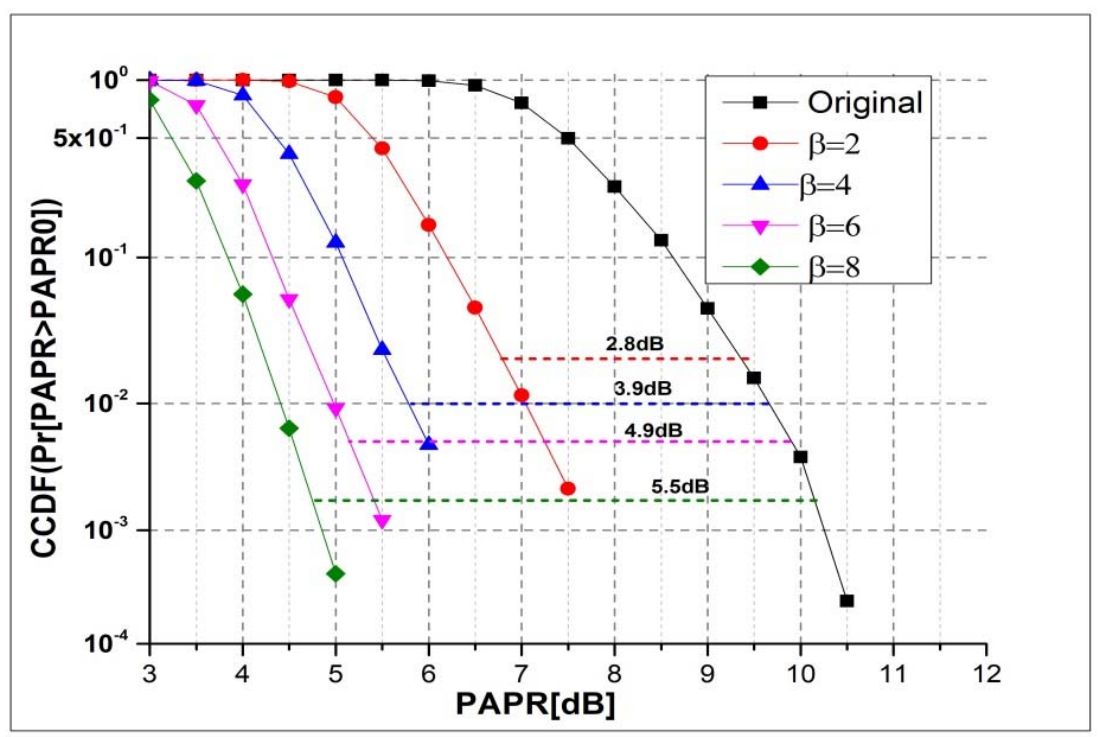

Fig.4. PAPR FOR DifFERENT VALUES OF B , $\mathrm{A}=0.2$, NSC $=128$, 4QAM

Figure(5) represents a comparison of the values of PAPR. The value of PAPR for the original signal and its value when using the clipping algorithm or the proposed algorithm MSNT is to reduce PAPR. From this figure, it will be possible to notice that the PAPR of the clipping, MSNT algorithm 
supplies a reduction of $0.75 \mathrm{~dB}$ and $4.15 \mathrm{~dB}$, respectively, when the probability of the given threshold is $10^{-3}$.

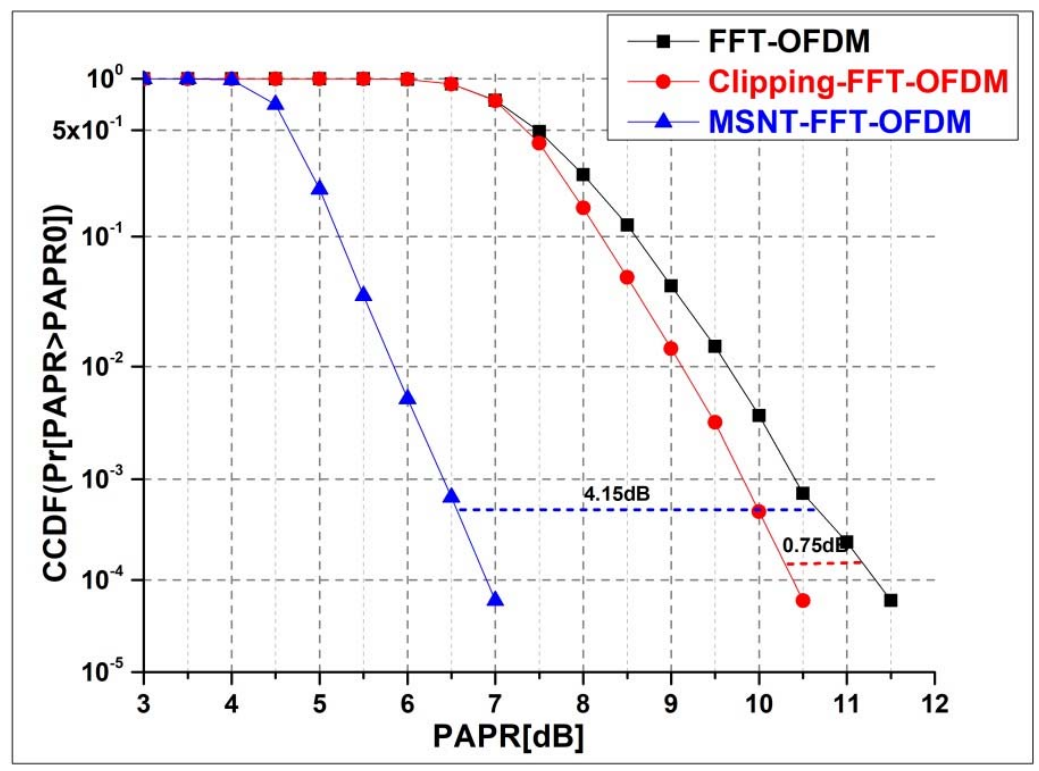

Fig. (5) CCDF FOR ORIGINAL SignAL, ClipPING AND MSNT AlgORITHM

Table (2) shows the value of PAPR in dB for different values of $(\alpha=0.2,0.4,0.6,0.8),(\beta=8)$, a different number of subcarriers and QAM order. This table shows that MSNT is less sensitive to the mapping order, but it is influenced by the number of subcarriers and the parameters $\alpha \& \beta$, which, if properly selected, we can get the lowest value of PAPR.

TABLE.2. PAPR VALUE FOR DIFFERENT VALUES OF NSC AND QAM ORDER

\begin{tabular}{|c|c|c|c|c|c|}
\hline \multicolumn{6}{|c|}{ PAPR in dB } \\
\hline \multirow[t]{2}{*}{ Nsc } & \multirow{2}{*}{ QAM } & \multicolumn{4}{|c|}{$\beta=8$} \\
\hline & & $\alpha=0.2$ & $\alpha=0.4$ & $\alpha=0.6$ & $\alpha=0.8$ \\
\hline \multirow{3}{*}{32} & 4 & 5.5 & 7.2 & 8.3 & 9.1 \\
\hline & 16 & 5.5 & 7.2 & 8.3 & 9.1 \\
\hline & 64 & 5.5 & 7.2 & 8.3 & 9.1 \\
\hline \multirow{3}{*}{64} & 4 & 6.2 & 7.5 & 8.2 & 8.8 \\
\hline & 16 & 6.2 & 7.5 & 8.2 & 8.8 \\
\hline & 64 & 6.2 & 7.5 & 8.2 & 8.8 \\
\hline \multirow{3}{*}{128} & 4 & 7.3 & 8.5 & 9 & 9.4 \\
\hline & 16 & 7.3 & 8.5 & 9 & 9.4 \\
\hline & 64 & 7.3 & 8.5 & 9 & 9.4 \\
\hline
\end{tabular}

Now we will investigate the performance of the system with and without the PAPR reduction algorithm in terms of bit error rate(BER), error vector magnitude(EVM), quality factor(QF) and constellation diagram.

Figure 6 illustrates the relation between EVM and the distance. The EVM increases by increasing the distance. EVM is indicating the quality of the QAM demodulation in the receiver regarding its 
ability to retrieve the signal. EVM for the system with and without the PAPR algorithm equals to 0.235 and 0.245 , respectively at $660 \mathrm{~km}$ with a reduction equals to $1 \%$.

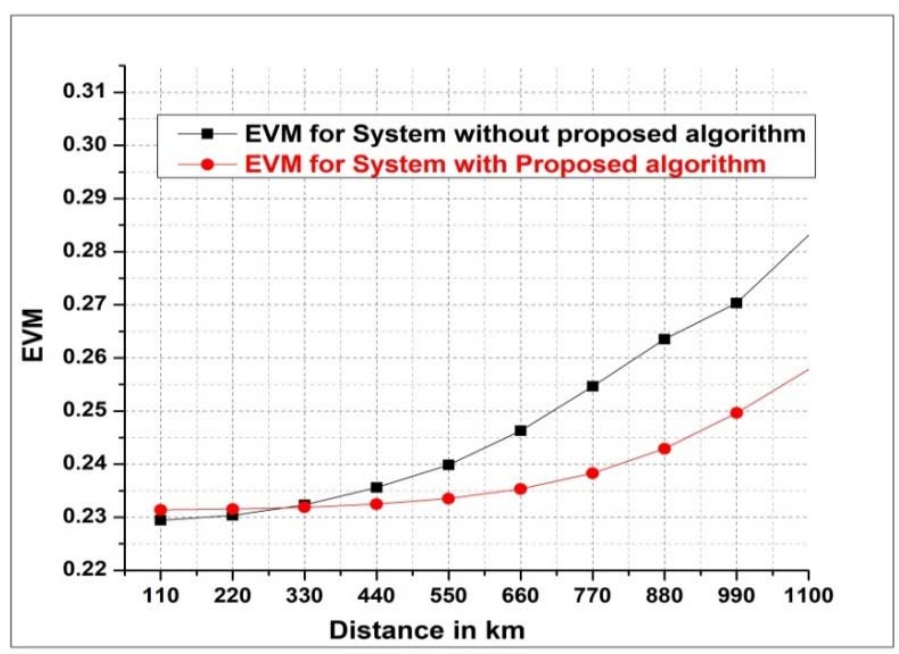

FIG. (6) EVM vs. DisTANCE IN KM

Figure 7 shows the relation between $\mathrm{QF}$ and the distance. The QF decreases by increasing the distance. QF is indicating the quality performance for the system and how the system could transfer the signal over long-haul distance. The quality factor at a short distance approaches to infinity because BER approaches to zero. Also, at a short distance, the amplifier and DCF could compensate the effect of attenuation, dispersion, and nonlinear impairments, when the distance increases the effect of these impairment increases too, which leads to degrading the QF. From the figure, the quality factor of the system with and without the PAPR algorithm at a distance of 770 equals to $8.12 \mathrm{~dB}$ and 2.91 , respectively, which indicates that the algorithm has improved the quality factor by $5.21 \mathrm{~dB}$.

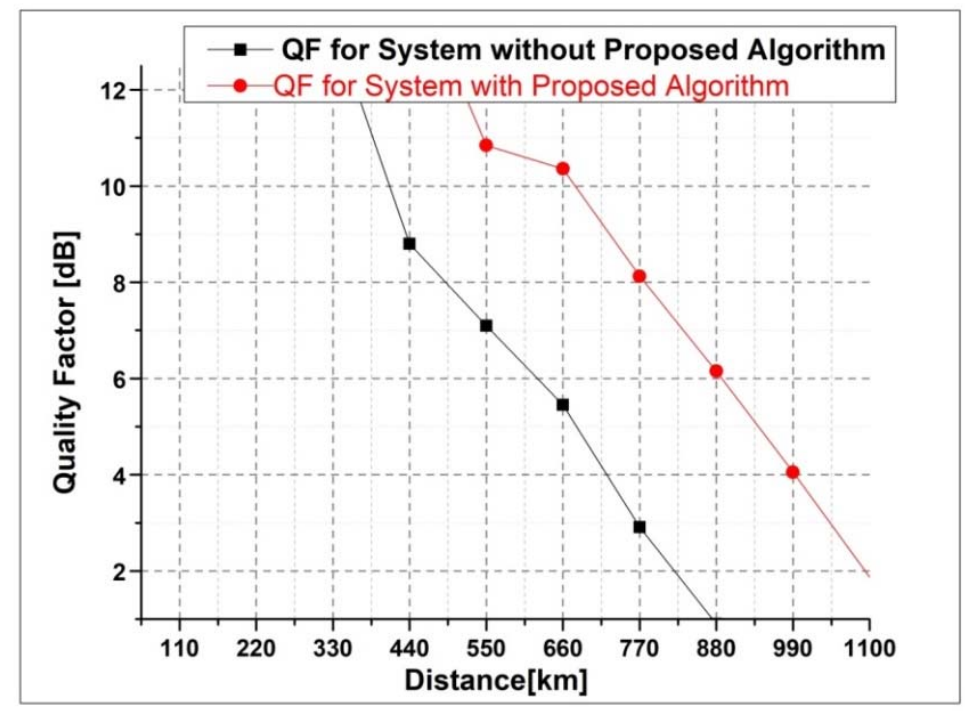

FIG. (7) QF VS. DISTANCE IN KM

Figure 8 shows the relation between BER and distance, where as the distance increases, the BER also increases because the power of the signal decreases. As previously explained, in short distances the BER approaches zero because an optical amplifier and DCF control the optical channel impairment. However, as the distance increases, the effect of impairments starts to appear, which means the need to 
increase the signal power in order to reach a long distance. Increasing power means that the PAPR is increasing too. From the figure, BER for the system with the PAPR algorithm reaches the limit of $10^{-3}$ at a distance of $660 \mathrm{~km}$, while the system without the PAPR algorithm reaches the limit at a distance of $395 \mathrm{~km}$. Therefore, there is an enhancement in the distance equals to $265 \mathrm{~km}$.

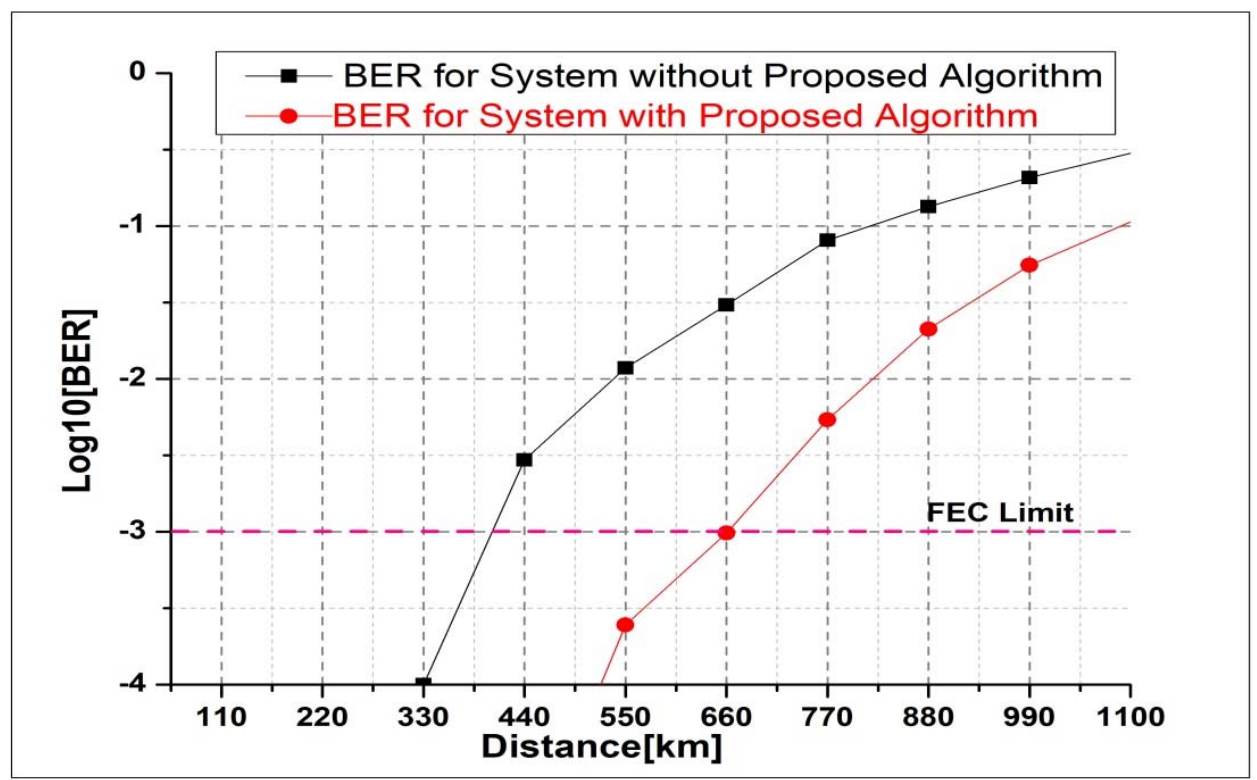

Fig. (8) BER VS. DiSTANCE IN KM

Figure $(9 \mathrm{a}, \mathrm{b})$ shows the constellation diagram for the signal at the receiver with and without adding the PAPR reduction technique at a distance equals to $770 \mathrm{~km}$ and quality factor equals to $8.1 \mathrm{~dB}$ and $3.1 \mathrm{~dB}$, respectively.

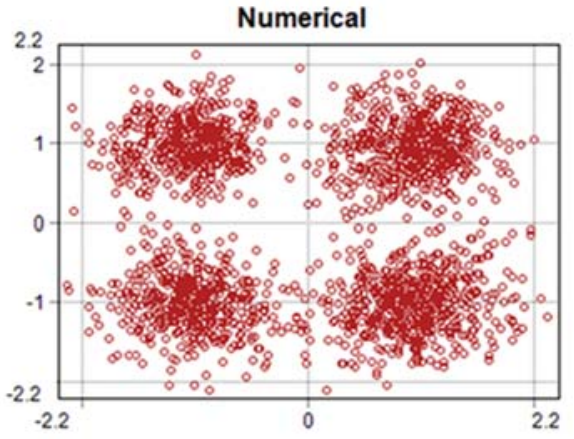

(a)

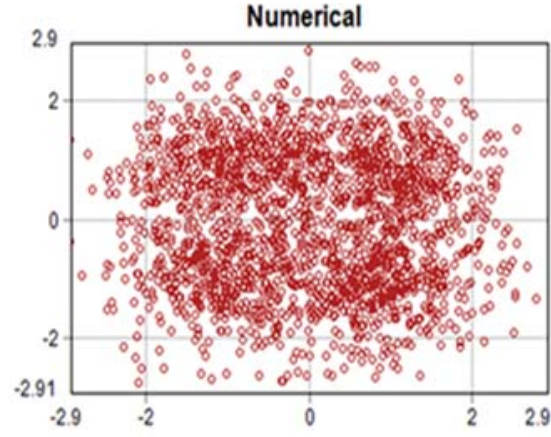

(b)

Fig. (9) Constellation Diagram a) With PAPR Reduction technique

B) WITHOUT PAPR REDUCTION TECHNIQUE

\section{Conclusion}

OFDM could be a promising technique for high data rate communication systems. Its advantages concern on high spectral efficiency and reduced ISI. However, high PAPR is the associate degree inherent downside of OFDM that arises due to an oversized range of subcarriers. Once the subcarriers are added coherently, the outcome signal will turn out a high peak power that affects the performance of the system. Therefore, many techniques are used to reduce PAPR of the OFDM signal. In this paper, a nonlinear method MSNT is used in a CO-OFDM system for the first time to mitigate PAPR. The graph of CCDF illustrates that the proposed technique offers higher PAPR reduction than using MSNT 
method. The reduction in PAPR achieves 4.15dB. Also, this technique has been investigated to examine the BER, Quality factor and constellation diagram when the fixed laser power is $10 \mathrm{dBm}$ rated at $10 \mathrm{Gbps}$ SMF over an allowable distance up to $770 \mathrm{~km}$. The obtained results have shown that the proposed technique has led to a very significant improvement in the system performance.

\section{REFERENCES}

[1] J. Armstrong, "OFDM for optical communications," Journal of lightwave technology, vol. 27, pp. 189-204, 2009.

[2] S. PROCESSING. (2015). OFDM transmitter bandwidth. Available: https://dsp.stackexchange.com/questions/20132/ofdm-transmitter-bandwidth

[3] W. Shieh and C. Athaudage, "Coherent optical orthogonal frequency division multiplexing," Electronics Letters, vol. 42, pp. 587-589, 2006.

[4] O.-J. Kwon and Y.-H. Ha, "Multi-carrier PAP reduction method using sub-optimal PTS with a threshold," IEEE Transactions on Broadcasting, vol. 49, pp. 232-236, 2003.

[5] Z.-r. Tong, Y.-n. Hu, and W.-h. Zhang, "PAPR reduction in CO-OFDM systems using IPTS and modified clipping and filtering," Optoelectronics Letters, vol. 14, pp. 209-211, 2018.

[6] S. Kaur and G. S. Saini, "Review paper on PAPR reduction techniques in OFDM system," Indian Journal of Science and Technology, vol. 9, 2017.

[7] D. Agarwal, N. Sharan, M. P. Raja, and A. Agarwal, "PAPR reduction using precoding and companding techniques for OFDM systems," in Computer Engineering and Applications (ICACEA), 2015 International Conference on Advances in, 2015, pp. 18-23.

[8] T. H. Ali and A. Hamza, "A new SLM technique based on Genetic Algorithms for PAPR reduction in OFDM systems," International Journal of communications, 2016.

[9] C. Ye, Z. Li, T. Jiang, C. Ni, and Q. Qi, "PAPR reduction of OQAM-OFDM signals using segmental PTS scheme with low complexity," IEEE Transactions on Broadcasting, vol. 60, pp. 141-147, 2014.

[10] G. Sikri, "Rajni, "A Comparison of Different PAPR Reduction Techniques in OFDM Using Various Modulations," International Journal of Mobile Network Communications \& Telematics (IJMNCT), vol. 2, pp. 53-60, 2012.

[11] J.-C. Chen and C.-P. Li, "Tone reservation using near-optimal peak reduction tone set selection algorithm for PAPR reduction in OFDM systems," IEEE Signal Processing Letters, vol. 17, pp. 933-936, 2010.

[12] M. Niranjan and S. Srikanth, "Adaptive active constellation extension for PAPR reduction in OFDM systems," in Recent Trends in Information Technology (ICRTIT), 2011 International Conference on, 2011, pp. 1186-1189.

[13] T. Jiang and G. Zhu, "Complement block coding for a reduction in a peak-to-average power ratio of OFDM signals," IEEE Communications Magazine, vol. 43, pp. S17-S22, 2005.

[14] Z.-p. Wang, J.-n. Xiao, F. Li, and L. Chen, "Hadamard precoding for PAPR reduction in optical directdetection OFDM systems," Optoelectronics Letters, vol. 7, p. 363, 2011.

[15] P. Singh, "Review of Non-Linear Transform based Techniques to Reduce the PAPR in OFDM Systems," International Journal of Computer Applications, vol. 95, 2014.

[16] R. Salmanzadeh and B. M. Tazehkand, "A modified method based on the discrete sliding norm transform to reduce the PAPR in OFDM systems," ETRI Journal, vol. 36, pp. 42-50, 2014.

[17] J. Panwar and Y. Jain, "Clipping Technique for BER and PAPR Reduction in OFDM System," International Journal Of Engineering And Computer Science, vol. 4, 2015.

[18] P. Gupta, B. A. Kumar, and S. K. Jain, "Peak to average power ratio reduction in OFDM using higher order partitioned PTS sequence and Bose Chaudhuri Hocquenghem Codes," in Signal Processing And Communication Engineering Systems (SPACES), 2015 International Conference on, 2015, pp. 443-447.

[19] S. Dursun and A. M. Grigoryan, "Nonlinear L2-by-3 transform for PAPR reduction in OFDM systems," Computers \& Electrical Engineering, vol. 36, pp. 1055-1065, 2010.

[20] D. Gupta and D. Dhawan, "A Study of Various PAPR Reduction Techniques for Optical OFDM Systems," International Journal of Computer Applications, vol. 115, 2015. 